\title{
Indwelling Urinary Catheter Placement and Removal Practices among Surgical Patients at Kigali University Teaching Hospital
}

\author{
Joselyne Mukantwari ${ }^{1 *}$, Lilian Omondi ${ }^{1}$, Donatilla Mukamana ${ }^{1}$, Oluyinka Adejumo ${ }^{1}$ \\ ${ }^{1}$ Department of General Nursing, School of Nursing and Midwifery, College of Medicine and Health Sciences, University of Rwanda
}

*Corresponding author: Joselyne Mukantwari. Department of General Nursing, School of Nursing and Midwifery, College of Medicine and Health Sciences, University of Rwanda, Remera Campus, KG 11 Ave, 47, Kigali, Rwanda. Email:mujoselyne@gmail.com

\begin{abstract}
Background

Indwelling urinary Catheters (IUCs) are routinely used medical devices among patients undergoing surgery and their undue use is a risk factor for urinary tract infections (UTIs). The study aim was to assess IUCs placement and removal practices among patients undergoing surgery.

Methods

We carried out a cross-sectional study on 207 patients operated at Kigali University Teaching Hospital (KUTH). A pretested observation checklist (Cronbach: $\alpha=0.851)$ was used for data collection. The data were analyzed using SPSS version 20. Chi-square test was used to determine the association between patients' characteristics and IUCs placement and removal.

Results

The IUC placement rate was 56.5\% ( $\mathrm{n}=117)$. There was significant association of IUCs use with gender, age, type and urgency of surgical procedures performed and the type of anesthesia used. Appropriate indication for IUC placement was established in 95.7\% patients $(n=112)$. Although $99.1 \%(n=116)$ patients were discharged from theatre with IUCs in situ, only $56.5 \%$ $(n=66)$ had documented removal instructions.

Conclusion

This study established that IUCs use is common and justified in surgery. However, lack of removal instruction was found to be a challenge. Therefore, the authors recommend patients' re-evaluation for post-surgery IUC need and its early removal where inappropriate to prevent CAUTIs and other complications.

Rwanda J Med Health Sci 2019;2(3):265-271.
\end{abstract}

Keywords: Indwelling urinary catheter use, surgical patients, surgery

\section{Introduction}

Urinary catheters are medical devices that are mostly used in critical care services [1] including operating theatres. About $15 \%$ to $25 \%$ of hospitalized patients, with up to $100 \%$ in the critical care unit, are exposed to urinary catheterization.[2,3] Most of the urinary catheterizations $(21 \%$ to $63 \%)$ are done without proper indications or placed for appropriated indications but unnecessarily prolonged.[4]

The catheter-associated urinary tract infections (CAUTIs) are considered the most challenging healthcare-associated infections (HCAIs) worldwide.[1,5-7] IUCs placement contribute up to $40 \%$ of HCAIs,[2,7] corresponding to one million of CAUTIs each year.[4] An IUC is also contributes $80 \%$ of UTIs morbidity among hospitalized patients, [8] 48\% of antimicrobial resistance[8] with a related cost of US\$676 on admission and $\$ 2836$ when complicated by bacteraemia.[4] The CAUTIs bacteria are coupled with higher antimicrobial use and resistance. [9]
Urinary catheterization is mostly done as perioperative routine care for the safety, and monitoring of the effectiveness of surgery.[5] However, CAUTIs are the third most common postoperative infections in addition to surgical site infection and bloodstream infection among operated patients. [10] IUC was also linked with other non-infectious complications like urethra trauma, discomfort and immobility.[4,11,12]

Different interventions to prevent CAUTIs have been studied for their effectiveness including the use of different types of catheters [1,13-15] like antiseptic, antibiotic, and silver coated catheters among others.[1] However, none revealed significance in reducing CAUTIs. Thus, the non-use or appropriate use of urinary catheter has been found to be effective in reducing the incidences of CAUTIs. The effective strategies to prevent CAUTIs after surgery are no placement or immediately removed catheter.[16,17] They cut down the rate of CAUTIs(17\% to $65 \%) \cdot[18]$

The Centre for Disease Control (CDC) through 
the Healthcare Infection Control Practices Advisory Committee (HICPAC) provided guidelines for using appropriately an IUC to prevent and reduce the cost of CAUTIs. The main guideline is to avoid unnecessary use of a urinary catheter.[19] HICPAC issued indications for appropriate use of IUC.[20] CDC recommended perioperative IUC use in the following conditions: "(1) urologic surgery or other surgery on nearby structure of genitourinary tract, (2) predictable prolonged duration of surgery and this catheter has to be removed in PACU, (3) patients expected to receive large amount of fluids or diuretics during surgery".[11,21] For patients with urine retention, those with sacral or perineal wounds and are incontinent and those with the need for immobilization following surgery,[21] IUC placement should be performed under medical order and in theatre. CDC recommends that an IUC placement for any inappropriate reason should be removed immediately. $[21,22]$

In Rwandan hospitals, the IUCs are being used among hospitalized patients including those who are undergoing surgeries. The bacteria like Klebsiella, Proteus, Citrobacter, Pseudomonas aeruginosa were predominantly isolated in patients with IUC, hospitalized in two University teaching hospitals. The associated antimicrobial resistance varied from $98.9 \%$ for amoxicillin, $90.4 \%$ for trimethoprim-sulphamethoxazole, $74.6 \%$ for nalidixic acid, $72.3 \%$ for amoxicillin-clavulanic acid, $57.4 \%$ for ciprofloxacin, $54.3 \%$ for ceftriaxone, $53.2 \%$ for nitrofurantoin to $50 \%$ for ceftazidine and other antibiotics which are commonly used medications to treat UTIs in Rwanda.[9] However, there is no study conducted about the use of IUC in Rwandan hospitals especially among perioperative patients. This motivated the researchers to carry out this study.

\section{Methods}

The study took place at operating rooms of KUTH among patients operated between $21^{\text {st }}$ March and $21^{\text {st }}$ April 2017. A cross-sectional study design was used. The hospital admits patients referred from district hospitals for further evaluation, diagnosis and treatment. The hospital is located in Kigali, the capital city of Rwanda. This hospital has two operating theatres, main theatre and maternity theatre each with eight operating rooms. Both theatres operate 420 patients on average per month that constituted the study population. Patients undergo different types of surgery which may include general surgery, Gynaecology \& Obstetrics, Ear Nose Throat (ENT), Ophthalmology, Neurosurgery, Plastic and reconstructive Surgery, Orthopaedics and trauma surgery, vascular surgery, Urology, Oral and Maxillofacial surgery, paediatric surgery and sometimes thoracic surgery to exclude cardiac surgery. Major organ transplant surgery such as kidney, liver and heart was not yet available in the hospital.[23] The study recruited patients of all ages with different diagnoses, scheduled for emergency or elective surgery and whether the
IUC was inserted in theatre or in the ward for surgical purpose. Patients admitted to the operating theatre (OT) with an indwelling urinary catheter placed for other than surgical reasons were excluded from the study.

The sample size was estimated using Yamane Formula

$$
n=\frac{N}{1+N(e)^{2}}
$$
at a confidence limit of 95\% and accepted margin error of $0.05 . \mathrm{N}$ was the estimated number of patients expected to undergo surgery in both OTs of KUTH for a period of one month that was 420 patients. The estimated sample size was 205 participants. A proportional sample was conveniently selected in both OT.

The institutional review boards of the University of Rwanda and KUTH approved the study. Individual consents were signed by patients who were meeting the conditions for signing a consent, next of kin for unconscious adult patients, parents or guardians for under 18 years old children. Fourteen to 17 years old children signed an assent form while their parents or guardians signed a consent form.

A pre-established observation checklist adopted from Catheter out project developed by Michigan University and sponsored by Agency for Healthcare Research and Quality (AHRQ) [20] was used to collect data with permission. Minor modifications were made to adapt it for local use in the perioperative period. The tool was tested for reliability on 17 patients before data collection who did not participate in the real study. The study tool was reliable at a Cronbach's $\alpha$ of 0.851 . The data were analysed using the statistical Package for Social Sciences (SPSS) software version 20. Both descriptive statistics and inferential statistics were used. Descriptive statistics were used to display data in frequencies and percentages of the characteristics of participants, and prevalence of IUC use. A Pearson chi-square test and Fisher exact test were calculated to identify the association of participants' characteristics with IUC placement and removal. The patients who were not exposed to IUC during their surgery were filtered and excluded before analysing the IUC removal rate and removal instructions.

\section{Results}

Among the 207 patients sampled, 108 were admitted in the main theatre and 99 in the maternity theatre. The demographic characteristics of participants (Table 1) show that majority were females (58.45\%) and their mean age was 34.7 years $(\mathrm{SD}: \pm 17.7)$. The majority underwent emergent $(57.5 \%)$, major $(97.5 \%)$, and obstetrics \& gynaecology surgeries, under general anaesthesia $(58.5 \%)$ and regional anaesthesia (40.6\%). 
Table 1. Demographic characteristics of participants

\begin{tabular}{|c|c|c|c|}
\hline Variable & Value & Frequency & Percentage \\
\hline \multirow[t]{6}{*}{ Age } & $<15$ years & 17 & $8.2 \%$ \\
\hline & $15-25$ year & 31 & $15.0 \%$ \\
\hline & 26-35 years & 83 & $40.1 \%$ \\
\hline & $36-45$ years & 35 & $16.9 \%$ \\
\hline & 46-56years & 13 & $6.3 \%$ \\
\hline & 56years and above & 28 & $13.5 \%$ \\
\hline \multirow[t]{2}{*}{ Sex } & Female & 121 & $58.5 \%$ \\
\hline & Male & 86 & $41.5 \%$ \\
\hline \multirow[t]{7}{*}{ Type of Surgery performed } & Urology & 6 & $2.9 \%$ \\
\hline & \begin{tabular}{|l|} 
Obstetrics \& gynaecology \\
\end{tabular} & 65 & $31.4 \%$ \\
\hline & Orthopaedics & 34 & $16.4 \%$ \\
\hline & \begin{tabular}{|l|} 
Laparotomy \\
\end{tabular} & 35 & $16.9 \%$ \\
\hline & General & 39 & $18.8 \%$ \\
\hline & ENT & 16 & $7.7 \%$ \\
\hline & Neurology & 12 & $5.8 \%$ \\
\hline \multirow[t]{2}{*}{ Seriousness of surgery } & Minor & 4 & $2.5 \%$ \\
\hline & Major & 203 & $97.5 \%$ \\
\hline \multirow[t]{2}{*}{ Urgency of surgery } & Emergent & 119 & $57.5 \%$ \\
\hline & Elective & 88 & $42.5 \%$ \\
\hline \multirow[t]{3}{*}{ Type of anaesthesia used } & Local anaesthesia & 2 & $1.0 \%$ \\
\hline & Regional anaesthesia & 84 & $40.6 \%$ \\
\hline & General anaesthesia & 121 & $58.5 \%$ \\
\hline Total & & 207 & $100 \%$ \\
\hline
\end{tabular}

Regarding the prevalence of IUC, 117 patients out of 207 participants $(56.5 \%)$ were exposed to IUC and $80.3 \%$ of them were catheterized in operating room. A Cross tabulation and calculated chi-square test helped to identify the characteristics significantly associated with IUC placement to include age $(\mathrm{P}<0.001)$, gender $(\mathrm{P}<0.001)$, type of surgery $(\mathrm{p}<0.001)$, Seriousness of surgery $(p<0.001)$, Urgency of surgery $(p<0.001)$ and Type of anaesthesia used $(\mathrm{P}=0.002)$.

The IUC placement rate was as high as $78.3 \%$ in $26-$ 35 years group participants, $71.9 \%$ in female, $93.8 \%$ in patients operated for obstetric and gynaecologic conditions, $73.1 \%$ in emergent surgeries and $70.2 \%$ in patients operated under regional anaesthesia.

Table 2. Prevalence of IUC placement, association with IUC Placement with characteristics

\begin{tabular}{|c|c|c|c|c|c|c|c|}
\hline \multicolumn{2}{|c|}{ Demographic characteristics, } & \multirow{3}{*}{$\begin{array}{l}\mathbf{N} \\
\\
17 \\
\end{array}$} & \multicolumn{2}{|c|}{ IUC Placement } & \multicolumn{3}{|c|}{$\begin{array}{c}\text { Association with IUC } \\
\text { placement }\end{array}$} \\
\hline & & & $\begin{array}{r}\text { IUC placed } \\
n(\%)\end{array}$ & $\begin{array}{r}\text { IUC not placed } \\
n(\%)\end{array}$ & $\mathbf{X}^{2}$ & df & sig \\
\hline \multirow{6}{*}{ Age } & $<15$ years & & $1(0.9)$ & $16(94.1)$ & & & \\
\hline & $15-25$ year & 31 & $15(48.4)$ & $16(51.6)$ & & & \\
\hline & $26-35$ years & 83 & $65(78.3)$ & $18(21.7)$ & & & \\
\hline & $36-45$ years & 35 & $16(45.7)$ & $19(54.3)$ & & & \\
\hline & 46-56years & 13 & $6(46.2)$ & $7(53.8)$ & & & \\
\hline & 56years and above & 28 & $14(50)$ & $14(50)$ & 37.329 & 5 & $<0.001$ \\
\hline \multirow[t]{2}{*}{ Sex } & Female & 121 & $87(71.9)$ & $34(28.1)$ & & & \\
\hline & Male & 86 & $30(34.9)$ & $56(65.1)$ & 28.030 & 1 & $<0.001$ \\
\hline \multirow[t]{7}{*}{\begin{tabular}{|l|} 
Type of Surgery \\
\end{tabular}} & Urology & 6 & $4(66.7)$ & $2(33.3)$ & & & \\
\hline & Obst\&Gyn & 65 & $61(93.8)$ & $4(6.2)$ & & & \\
\hline & Laparotomy & 35 & $30(85.7)$ & $5(14.3)$ & & & \\
\hline & Orthopedic & 34 & $9(26.5)$ & $25(73.5)$ & & & \\
\hline & Neurosurgery & 12 & $8(66.7)$ & $4(33.3)$ & & & \\
\hline & General & 42 & $6(14.3)$ & $36(85.7)$ & & & \\
\hline & ENT+Maxillofacial surgery & 16 & 0 & $16(100)$ & 1.129 & 6 & $<0.001$ \\
\hline \multirow[t]{2}{*}{ seriousness of surgery } & Minor surgery & 5 & $2(40)$ & $3(60)$ & & & \\
\hline & Major surgery & 203 & $116(57.1)$ & $87(42.9)$ & 21.215 & 2 & $<0.001$ \\
\hline \multirow[t]{2}{*}{\begin{tabular}{|l|} 
Urgency of surgery \\
\end{tabular}} & Emergent & 119 & $87(73.1)$ & $32(26.9)$ & 31.341 & 1 & $<0.001$ \\
\hline & Elective & 88 & $30(34.1)$ & $58(65.9)$ & & & \\
\hline \multirow[t]{4}{*}{\begin{tabular}{|l|} 
Anaesthesia used \\
\end{tabular}} & Local anesthesia & 2 & $1(50)$ & $1(50)$ & & & \\
\hline & Regional anesthesia & 84 & $59(70.2)$ & $25(29.8)$ & & & \\
\hline & General anesthesia & 121 & $57(47.1)$ & $64(52.9)$ & 11.158 & 2 & 0.002 \\
\hline & Total & 207 & 117(56.5) & $\mathbf{9 0 ( 4 3 . 5 )}$ & & & \\
\hline
\end{tabular}


The study findings identified that $95.7 \%$ ( $\mathrm{n}=112$ out 117) of patients with IUC in place had an appropriate indication for IUC placement (Table3). Therefore, the majority of participants with IUC in place $(56.4 \%, \mathrm{n}=$ 66) did not have a documented removal instruction from the surgeon. Only $27.4 \%(n=32)$ had within 24 hours removal instruction and $12 \%(\mathrm{n}=14)$ had 24 hours to 48 hours removal instruction (Table 4). The surgeons recorded IUC removal instructions in patients' files as postoperative instructions, mostly in obstetric and gynaecological surgeries $(68.9 \%)$. The removal instructions were not documented in Orthopaedic, Neurologic and General surgeries (Table 4). Only one catheter in a patient who had undergone gynaecological surgery was removed immediately after surgery.

Table 3. Indications for IUC Placement among patients undergoing surgery

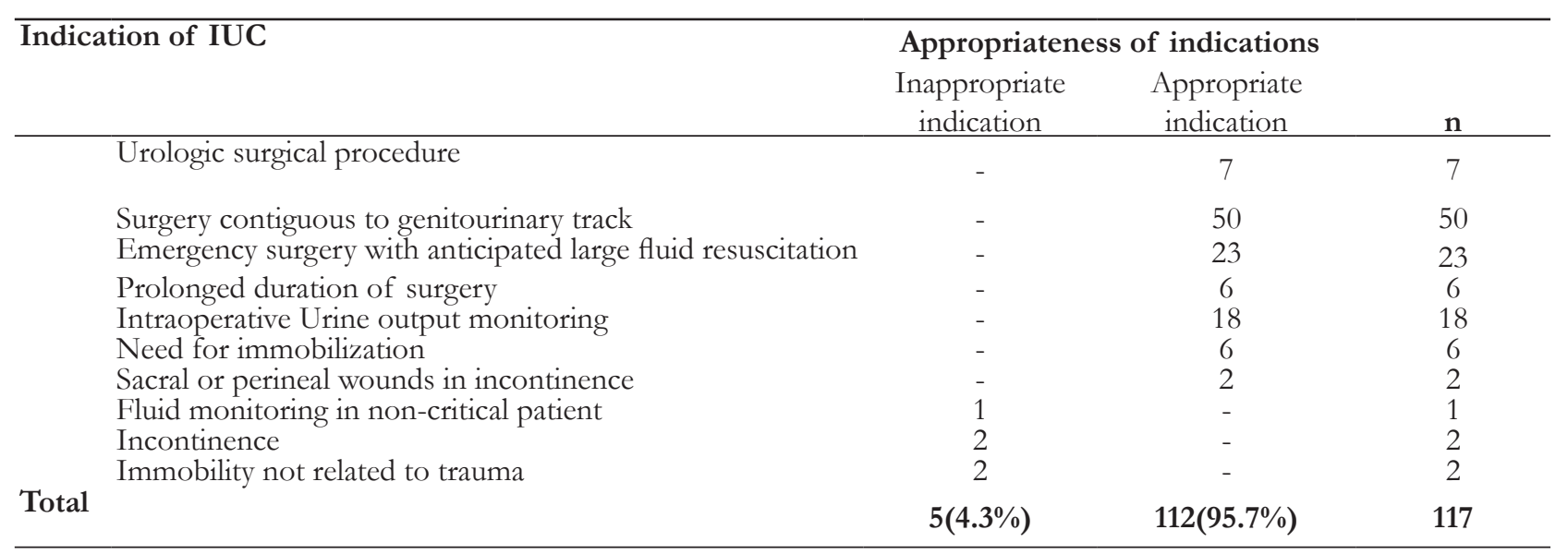

Table 4. Postoperative IUC removal instructions recorded by the surgeon

\begin{tabular}{|c|c|c|c|c|c|c|}
\hline \multirow{2}{*}{$\begin{array}{l}\text { Type of Surgery } \\
\text { performed } \mathrm{n}(\%)\end{array}$} & \multicolumn{5}{|c|}{ IUC removal instructions } & \multirow[b]{2}{*}{$\mathbf{N}$} \\
\hline & $\begin{array}{c}\text { Removal within } 24 \\
\text { hours }\end{array}$ & $\begin{array}{c}\text { Above } 24 \text { hours to } \\
48 \text { hours }\end{array}$ & $\begin{array}{c}\text { More } \\
\text { than } 48 \\
\text { hours (4) }\end{array}$ & $\begin{array}{c}\text { No removal } \\
\text { instructions (5) }\end{array}$ & $\begin{array}{c}\text { Any } \\
\text { Other (6) }\end{array}$ & \\
\hline Urology surgery & 0 & $1(25)$ & 0 & $3(75)$ & 0 & $4(100)$ \\
\hline Obst \& Gyn & $31(50.8)$ & $9(14.8)$ & 0 & $19(31.1)$ & $2(3.3)$ & $61(100)$ \\
\hline Laparotomy surgery & $1(3.3)$ & $4(13)$ & $1(3.3)$ & $22(73.3)$ & $2(6.7)$ & $30(100)$ \\
\hline Orthopedic surgery & 0 & 0 & 0 & $8(100)$ & 0 & $8(100)$ \\
\hline Neurologic surgery & 0 & 0 & 0 & $8(100)$ & 0 & $8(100)$ \\
\hline General surgery & 0 & 0 & 0 & $6(100)$ & 0 & $6(100)$ \\
\hline Total & $32(27.4)$ & $14(11.9)$ & $1(0.9)$ & $66(56.5)$ & $4(3.4)$ & 117 \\
\hline
\end{tabular}

\section{Discussion}

Starting with demographic characteristics of the study participants (Table1), almost three quarters $(73.3 \%)$ of the respondents were 15-45 years old. This is closely similar to national demographics [24] in which $71.6 \%$ of the total population is between 15-34 years old. Most of the participants were females in child bearing age and underwent obstetrics \& gynaecology surgeries (31.4\%) which were dominating surgical procedures in this study. In line of the leading surgeries, $(58.45 \%)$ were female whose surgery necessitated the use of urinary catheters to protect their pelvic organs.[25] Similarly, previous studies have shown the dominating number of women is surgery compared to men.[26,27] On the contrary, Walaszek[10] reported male dominance having studied neurosurgical patients only.
Besides Obstetrics \& Gynecology, other procedures performed included general surgeries, laparotomy surgeries and orthopedic surgeries. Most of these surgeries were classified major and emergent according to KUTH protocol of surgical classification. Being a teaching and referral hospitals, most of the time, the hospital admits complicated patients referred from smaller health care institutions. This is in agreement with the global distribution of surgeries that estimated the higher proportion of emergent obstetric surgeries in low-income countries, Rwanda included.[28] A study in East African countries[29] showed that the scope of surgical procedures undertaken are narrow while the emergent life-saving procedures and obstetric surgeries are the most commonly performed procedures. Another study[27] added to the list of abdominal surgeriesmainly laparotomies' and orthopedic surgeries especially 
of the extremities in tandem with this study.

Considering the the factors that guide the choice of anesthesia[30], general anaesthesia technique and spinal anaesthesia were the most used in this study. This conformed to anesthetic practices at KUTH in addition to patients' considerations and was similar to techniques reported in the previous study.[27] In contrast, other studies[26,31] found that the majority of participants were operated under spinal anesthesia $(70 \%)$, a technique that provides a reversible loss of sensation in a specific region or area [31].

This is attributed to the fact that their study was conducted among patients undergoing hip surgery and did not require patients to sleep. Similar to findings of other previous studies,[26,27] the IUC placement rate was significant associated with age, gender, type of surgical operation, seriousness and urgency of surgery and anesthesia technique used. Previously, a higher prevalence rate of IUC use has been reported in cardiac surgery.[32] Regional anesthesia has been associated with postoperative urine retention[33] hence the use of IUC postoperatively. Moreover, the study[27] found that among the patients operated in different surgical specialities, IUC was needed by $8.6 \%$ depending on their bladder capacity and $11.8 \%$ without considering individual bladder capacity.

The type, location and duration of the surgery determine the need for use or non-use of IUC. This justifies the routine use of IUC in some procedures such as obstetrics and gynaecology surgeries as well as in Laparotomies. IUCs are placed to empty bladder before pelvic surgery to prevent risks for bladder injuries and traumatizing other pelvic organs, to monitor input \& out and for fluid resuscitation in prolonged surgeries. In contrast, some studies have evidenced that IUC is not required in caesarean delivery, hysterectomy and other gynecologic procedures, if the patient is haemodynamically stable. $[34,35]$ Such patients are usually requested to void and empty bladder before surgery.

Although the duration of postoperative IUC retention and factors associated with non-immediate removal of the catheter were not explored within the scope of this study, prolonged duration of postoperative IUC was reported in previous studies to be associated with poor outcomes and complications.[34-37]. This study identified that the IUC removal instructions were not documented in $56.5 \%$ only and only $27.3 \%$ were recommended to be removed within 24 hours.

Further revelation from this study shows that only one $(0.9 \%)$ catheter was removed among 107 IUCs that were judged unnecessary in both general and maternity OTs based on available evidence, CDCs recommendations [21] and clinical status of the patients. However, immediate IUC removal after the procedure is recommended if the catheter is not required to remain in-situ in order to prevent postoperative CAUTIs and other related complications.[2,5,38,39] To prevent post-operative CAUTIs, the third most common postoperative infections, $[10,37]$ all perioperative patients care team should know and carefully apply the principles of aseptic and sterile techniques at all times during procedures.[40]

Although CDC recommends that perioperative IUC is used for a medical reason and not for routine or convenience of care,[20,21] most studies have not ventured to assess the preoperative medical diagnosis and the duration of surgery that may guide the surgeon in deciding the use or non use of IUC perioperatively.

\section{Conclusion and recommendation}

Indwelling urinary catheter is commonly used in surgical patients at KUTH and as a routine in some procedures such Obstetrics \& Gynecology, laparotomy, urology, neurologic, emergent surgeries, surgeries performed under spinal anesthesia, females and 2635 years old group. IUC placement was significantly associated with age, gender, type, seriousness and urgency of surgery, and type of anaesthesia used. The IUCs were placed for appropriate indications according to CDC guidelines such as surgery contiguous to genitourinary track, emergent surgery with anticipated large fluid resuscitation; intraoperative urine output monitoring and physician order was present in 61.5\% of placed IUCs. The IUC removal instructions were not documented in $56.5 \%$ of patient with catheter in situ, especially patients operated in orthopedic, laparotomy, neurosurgery, and general surgeries. Only 1IUC was removed before patients left the PACU. The IUC retention and related complications after surgery were not assessed in this study. Again, additional information on the patients' conditions, duration of surgery and the specific procedure guidelines for IUC use still needs to be investigated. Therefore, the authors recommend patients' re-evaluation for post-surgery IUC need and its early removal where appropriate to prevent CAUTIs and other complications.

\section{Limitations}

The study was conducted in one hospital, hence limiting the generalizability of the findings. The gravity of diagnosis and the time required by surgery determine the need for IUC; unfortunately, they were not assessed in the scope of this study.

\section{Acknowledgements}

We convey our gratitude and special thanks to the University of Rwanda especially the College of Medicine and Health Sciences for review and approval of this research, and Michigan University that allowed us to use and edit their study tool. 
We would like to acknowledge the patients, surgical teams and administrative body of Kigali University Teaching Hospital for their cooperation during this study.

\section{Conflict of interest}

The authors declare no conflicts of interest.

\section{Authors' contribution}

The authors contributed in the conception of the study framework, writing the research proposal, developing and validating the study tool, conducting or guiding data collection and analysis and development of the manuscript for publication.

This article is published open access under the Creative Commons Attribution-NonCommercial NoDerivatives (CC BY-NC-ND4.0). People can copy and redistribute the article only for noncommercial purposes and as long as they give appropriate credit to the authors. They cannot distribute any modified material obtained by remixing, transforming or building upon this article. See https://creativecommons.org/ licenses/by-nc-nd/4.0/

\section{References}

1. Lam, Omar, Fisher, Gillies, Maclennan. Types of indwelling urethral catheters for short-term catheterisation in hospitalised adults ( Review ). Cochrane Rev. 2014;1:1-92.

2. Andrade V, Fonseca L, Fernandes F, Veludo A. Prevention of catheter-associated urinary tract infection: implementation strategies of international guidelines. Review. Rev Latino-Am Enferm. 2016;24:1-9.

3. Jain M, Dogra V, Mishra B, Thakur A, Loomba PS. Knowledge and attitude of doctors and nurses regarding indication for catheterization and prevention of catheter-associated urinary tract infection in a tertiary care hospital. Indian J Crit Care Med. 2015;19:76-81.

4. Meddings J, Krein SL, Fakih MG, Olmsted RN, Saint S. chapter 9:Reducing Unnecessary Urinary Catheter Use and Other Strategies To Prevent Catheter-Associated Urinary Tract Infections: Brief Update Review. Mak Heal Care Safer II an Updat Crit Anal Evid Patient Saf Pract. Bethesda: National Center for Biotechnology Information, U.S. National Library of Medicine; 2014. p. 1-18.

5. Abdel-Aleem H, Aboelnasr M, TM J, Habib F. Indwelling bladder catheterisation as part of intraoperative and postoperative care for caesarean section ( Review ). TheCochrane Libr. 2014;1:1-42.

6. Amine AEK, Omar M, Helal M, Mohamed W, Bakr K. Evaluation of an intervention program to prevent hospital-acquired catheter-associated urinary tract infections in an ICU in a rural Egypt hospital Evaluation eines Interventionsprogramms zur Prävention nosokomialer. GMS Hyg Infect Control. 2014;9:1-10.

7. Conway LJ, Larson EL. Guidelines to prevent catheterassociated urinary tract infection: 1980 to 2010 . Hear Lung Author manuscript; 2012;41:271-83.

8. Lindsay N. Catheter associated urinary tract infections. Review. Antimicrob Resist Infect Control 2014,. 2014;3:1-8.

9. Muvunyi CM, Masasaisa F, Bayingana C, Mutesa L, Musemakweri A. Antimicrobial Resistance and Extended-Spectrum $\beta$ - Lactamase (ESBL) Producing Clinical Isolates from Urinary Tract Infection at two teaching hospitals in Rwanda. Rwanda Journal, Ser F Heal Sci. 2013;1:3-16.

10. Wałaszek M. The analysis of the occurrence of nosocomial infections in the neurosurgical ward in the district hospital from 2003 to 2012. PRZEGL Epidemiol. 2015;1:507-14.

11. Meddings J, Rogers MAM, Krein SL, Fakih MG, Olmsted RN, Saint S. Reducing unnecessary urinary catheter use and other strategies to prevent catheter-associated urinary tract infection: an integrative review. BMJ Qual Saf. 2014;23:277-89.

12. CDC. Urinary Tract Infection (Catheter-Associated Urinary Tract Infection [ CAUTI ] and NonCatheter-Associated Urinary Tract Infection [UTI]) and Other Urinary System Infection [USI ]) Events. Device-associated Modul UTI. 2015;1:7-16.

13. Jahn P, Beutner K, Langer G. Types of indwelling urinary catheters for long-term bladder drainage in adults ( Review ). 2012;

14. Jamison J, Maguire S, Mccann J. Catheter policies for management of long term voiding problems in adults with neurogenic bladder disorders ( Review ). cobyTheCochrane Collab. 2013;1-14.

15. So K, Habashy D, Doyle B, Chan L. Indwelling Urinary Catheters Pattern of Use in a Public Tertiary-level Australian Hospital. Urol Nurs. 2014;34:69-73.

16. Fakih MG, Krein SL, Rn BE, Msa SRW, Battles JB, Saint S. Engaging health care workers to prevent catheter-associated urinary tract infection and avert patient harm. Am J Infect Control. Elsevier Inc; 2014;42:S223-9.

17. Lo E, Lindsay N, Susan C, Gould C, Maragakis L, Meddings J, et al. Strategies to Prevent CatheterAssociated Urinary Tract Infections in Acute Care Hospitals: 2014 Update. Infect Control Hosp Epidemiol. 2014;35.

18. Xie AD, Lai R, Nie S, Health P, Control I, Hospital 
T, et al. Surveys of catheter-associated urinary tract infection in a university hospital intensive care unit in China. Braz J Infect Dis. 2011;15:296-7.

19. Conway L, Pogorzelska M, Larson E, Stone P. adoption of policies to prevent catheter-associated urinary tract infections in US intensive care unit. Amercan J Infect Control. 2013;40:705-10.

20. Fakih MG, Saint S, Manojlovich M, Krein S, Olmsted R. Appropriate urinary catheter placement in the emergency department. Agency Healthc. Res. Qual. (AHRQ). Agency for Healthcare Research and Quality (AHRQ).; 2012. p. 13-31.

21. Gould C V, Umscheid CA, Agarwal RK, Kuntz G, Pegues DA, Patrick J. Guideline for prevention of Catheter associated urinary tract Incections 2009. Atlanta, Philadelphia: Health care Infection Control Practices Advisory Commitee (HICPAC), CDC; 2009. p. 1-67.

22. Hu F, Yang D, Huang C, Chen C, Chang C. Inappropriate use of urinary catheters among hospitalized elderly patients : Clinician awareness is key. Geriatr Gerontol Int. 2014;1:1-7.

23. Kigali university teaching Hospital. Clinical Services \& Specialties. Overview. 2019. p. 1.

24. National Institute of Statistics of Rwanda. Demographic and Health Survey Rwanda[DHS] 2014/2015. Key findings Rwanda. kigali, Rwanda: MOH Rwanda; 2015. p. 1-29.

25. Abdel-Aleem H, Aboelnasr M, Jayousi T, Habib F. Indwelling bladder catheterisation as part of intraoperative and postoperative care for caesarean section ( Review ). Cochrane Database Syst Rev I. 2014;1:1-43.

26. Nymana MH, Gustafsson M, Langius-Eklof A, Johansson J-E, Norlin R, Hagberg L. Intermittent versus indwelling urinary catheterisation in hip surgery patients: A randomised controlled trial with cost-effectiveness analysis. Int J Nurs Stud. 2013;50:1589-98.

27. Brouwer TA, Rosier PFWM, Moons KGM, Zuithoff NPA, Roon EN van, Kalkman CJ. Postoperative Bladder Catheterization Based on Individual Bladder Capacity. A Randomized Trial. Anesthesiology. 2015;1:46-54.

28. Weiser TG, Haynes AB, Molina G, Lipsitz SR, Esquivel MM, Uribe-Leitz T, et al. Size and distribution of the global volume of surgery in 2012. Bull World Health Organ. 2016;94:201-209F.

29. Galukande M, von Schreeb J, Wladis A, Mbembati $\mathrm{N}$, de Miranda H, Kruk ME, et al. Essential surgery at the district hospital: A retrospective descriptive analysis in three african countries. PLoS Med. 2010;7:1-10.
30. Rothrock J, McEwen D. Alexander's Care Of the Patient in Surgery. 15th Editi. McEwen D, editor. Elsevier Inc; 2015.

31. Rothrock JC. Alexander' care of the patient in surgery. 15th editi. McEwen D, editor. liverport: Elsevier MOsby Inc; 2015.

32. Conterno LO, Martins S, Toni D, Konkiewitz RG, Guedes ES, Barros RT De, et al. Impact of hospital infections on patients outcomes undergoing cardiac surgery at Santa Casa de Misericórdia de Marília. Rev Bras Cir Cardiovasc. 2014;29:167-76.

33. Ryamukuru D, Mukantwari J, Omondi L, Karonkano GR, Munyaneza E, Karera E, et al. Clinical experiences of perioperative nursing Masters Students in selected Rwandan Referral Hospitals. Rwanda J Med Heal Sci. 2018;1:34-8.

34. Bharti J, Aggarwal N, Chopra S, Taneja N. A prospective randomized controlled comparison of immediate versus late removal of urinary catheter after abdominal hysterectomy. J Midlife Health. 2014;5:68-71.

35. Pandey D, Mehta S, Grover A, Goel N. Indwelling catheterization in caesarean section: Time to retire it! J Clin Diagnostic Res. 2015;9:QC01-4.

36. Alvarez AP, Demzik AL, Alvi HM, Hardt KD, Manning DW. Risk Factors for Postoperative Urinary Tract Infections in Patients Undergoing Total Joint Arthroplasty. Adv Orthop. 2016;2016:1-5.

37. Nancy MP. Berry \& Kohn's Operating Room Technique. Thirteenth. liverport: Elsevier; 2017.

38. Revello K, Gallo A. Implementing an evidencebased practice protocol for prevention of catheterized associated urinary tract infections in a progressive care unit. J Nurs Educ Pract. 2013;3:99-107.

39. Gould C V, Umscheid C a, Agarwal RK, Kuntz G, Pegues D a, Patrick J. Guideline for P Revention of Catheter - aasociated Urinary Tract Infections 2009. guidelines. Atlanta: CDC; 2010. p. 1-67.

40. Fakih MG, Watson SR, Greene MT, Kennedy EH, Olmsted RN, Krein SL, et al. Reducing Inappropriate Urinary Catheter Use, A Statewide Effort. Arch Intern Med. 2012;172:255. 